\title{
Modified Surgical Technique for Combined Congenital External and Middle Ear Malformations
}

\author{
Milan Stankovic, Sasa Zivaljevic, \\ Dragana Simic, Bojan Marinkovic, \\ ENT Clinic Nis, University of Nis, Serbia
}

Short running head: congenital malformations of ear Author: Milan Stankovic, ORL Clinic, Medical Faculty Nis, Serbia

\begin{abstract}
:
Hypothesis: There is a positive correlation between type and intensity of combined congenital ear malformations and results of surgical reconstruction.

Background: Combined congenital malformations of external and middle ear pose a lot of surgical problems and demand adequate skill and planned, fractioned therapy. The goals of these operations are: limited number of operations with minimal complications, well cosmetic result, hearing improvement and easier use of hearing aid, removal of congenital cholesteatoma, preservation of labyrinth and facial nerve.

Methods: The aim of this work is to present the experience in treating patients with combined malformations of external and middle ear. In the period between 1992 and 2001 seventeen patients with this type of congenital malformations were operated. Classical dilemma who operates first, plastic surgeon or otosurgeon is solved here by complete otosurgical treatment with reduced number of operations.

Results: Totally 36 operation was performed, two reoperations were made in four patients, and also three operations in three patients for the reconstruction of deformity of auricle. In seven patients costal rib cartilage was used for auricular framework. Reoperations were done during the course of auricular reconstruction (14 operations), or because of meatal restenosis (five operations), or cartilage graft necrosis (one case). Atresia was adequately solved in 14 patients, while in three cases connective tissue meatal restenosis was found. The results of reconstruction of auricular malformation were aesthetically good in 13 patients and sufficient in four patients.

Conclusion: We found positive correlation between external ear and middle ear deformity, and the results of reconstruction, as well. Two thirds of patients have hearing threshold level under 30 $\mathrm{dB}$, while the threshold in other patients is $30-40 \mathrm{~dB}$. Residual airbone gap amounting about $20 \mathrm{~dB}$ was universally present.
\end{abstract}

Key words: Congenital malformations, External ear, Middle Ear, Surgery.

\section{Introduction}

The first written traces concerning ear malformations are found in ancient era. However, the first confirmed description of ear malformations date in VII century when Paulus (form Aegina) mentioned the differences between congenital and acquired forms. Treatment consisted of incision and cauterisation. Scientifically based reconstruction was tried in 1920 when Gillies used costal cartilage graft. Different materials for restoration of delicate auricular shape were used, such as cobalt-chrome mash, acrilate, silastic and composite grafts. The reasons for many materials and techniques are insufficient aesthetic results and many operations needed to achieve good outcome when costal cartilage is used. During the last 50 years development of tympanoplasty technique enabled essential changes in treatment of combined malformations of external and middle ear. Besides this, new radiologic and audiologic diagnostic methods led to proper selection and preparation of these patients for surgical reconstruction. ${ }^{1-2}$

Embryonal development of auricle includes auricular tubercles derived from mandibular (the first three) and hyoid arch (the last three). External auditory canal and tympanic membrane are formed from the first branchial pouch. The first and the second branchial arch are important for formation of auditory ossicles, excluding stapedial basis. Tympanic part of temporal bone also calcifies postnatally creating medial part of external meatus. Meatal atresia is caused by irregularities of this process. Cartilaginous atresia is possible, even if tympanic ring and tympanic membrane are developed. ${ }^{1-2}$

Different classification of ear malformations, mainly descriptive can be found. The simplest one is on big and little deformities. Big deformities include marked microtia, underdevelopment of meatus and tympanic membrane, reduced middle ear space, and deformed and fixed auditory ossicles. On the other side, small deformi- 
ties are characterised by moderate or little deformity of ossicles, regular meatus and pneumatic middle ear space. Auricular malformations can be classified as little (type I), vertical rudiment of auricle (type II), and small auricular remnant (type III). ${ }^{1}$

Combined malformations of both structures are very important and difficult to solve. Other deformities, particularly affecting mandible, can be also found. Facial canal malformations, such as dehiscence or irregular course are frequent in combined forms. Auditory ossicles are often fused, with smaller, deformed malleus and other changes. Reduced pneumatisation indicates on changes of other middle ear structures. As inner ear derives from ectodermal otocyst, combined malformations of middle and inner ear are rare.

The aim of this work is to present our experience in surgical treatment of congenital, mixed deformities of external and middle ear, and to present characteristics of specific type of reconstruction that was applied.

\section{Material and Methods}

Complete ENT examination, auditory evoked potentials and computerised tomography were routinely performed preoperatively. During the ten years period (1992-2001) 17 patients were operated because of congenital external and middle ear malformations. There were seven mayor ear malformations, six atresia and middle ear deformities, and four minor external and middle ear malformations. Totally 33 operations were performed.

Classical surgical procedure for reconstruction of combined external and middle ear malformations has few phases. In order to improve the results of reconstruction and to avoid dilemma: who operates first - plastic surgeon or otosurgeon; we modified the timing, order and type of surgical interventions. In the first phase reconstruction of atresia and middle ear space, with implantation of cartilaginous transplant is performed. Costal rib cartilage is shaped according to prepared model. This operative phase is the longest, and the most complex. It is performed completely by otosurgeon. In the next phase auricle is elevated, and in final stage precise reconstruction of certain auricular parts is done (table 1 and 2).

Table 1. Integrated Auricular Reconstruction Protocol (IARP) Aguilar EF (1996)

\begin{tabular}{ll}
\multicolumn{1}{c}{ STAGE AND TYPE OF OPERATION } & \multicolumn{1}{c}{ SURGEON } \\
$\begin{array}{l}\text { Stage I Harvesting and placement of } \\
\text { cartilaginous frame }\end{array}$ & Plastic surgeon \\
Stage II Creation of lobule & Plastic surgeon \\
Stage III Atresia repair & Otosurgeon \\
Stage IV Creation of tragus & Plastic surgeon \\
Stage V Elevation of auricle & Plastic surgeon \\
\hline
\end{tabular}

Table 2. Modified surgical technique for atresia and microtia repair

STAGE AND TYPE OF OPERATION

Stage I Harvesting and placement of cartilaginous frame. Atresia repair

Stage II Creation of lobule and tragus

Stage III Elevation of auricle, minor corrections

For reconstruction of meatal atresia and middle ear space anterior incision is used with elevation of soft tissue in two layers. Skin incision should never be placed over cartilaginous transplant. Atretic plate is visualized, completely removed with burr. Malformed ossicles are inspected, their mobility, shape and relation with other structures are analysed. After surgical enlargement of external meatus and middle ear space, adequately big temporalis fascia is placed over ossicular system and borders of created anulus. Meatoplasty with resection of soft tissues, and formation of new canal with $50 \%$ bigger diameter than usual are next step. Proper position of auricle and cartilaginous canal is assured.

Reoperations were done for staged reconstruction of auricle, or for correction of inadequate anatomic or functional outcome. Aesthetic and audiologic postoperative results for at least six months were analysed.

\section{Results}

Applied surgical technique resulted in adequate diameter of meatus in $61,5 \%$, while in $38,5 \%$ restenosis was found. Reoperations were performed in cases of restenosis, and only in one case $(7,7 \%)$ surgical result was insufficient. Postoperatively in four patients infection of skin was verified, but after parenteral and local antibiotic treatment it was eliminated (table 3. and 4.).

Table 3. Type of deformity and number of performed operations

\begin{tabular}{lclcc}
\multicolumn{1}{c}{ MIDDLE } & CANAL & AURICLE & \multicolumn{2}{c}{ NUMBER } \\
EAR & patients & operations \\
$\begin{array}{l}\text { Big } \\
\text { deformity }\end{array}$ & Atresia & $\begin{array}{l}\text { Vertical } \\
\text { rudiment }\end{array}$ & 7 & 24 \\
$\begin{array}{l}\text { Small } \\
\text { deformity }\end{array}$ & Atresia & $\begin{array}{l}\text { Moderate } \\
\text { deformity }\end{array}$ & 6 & 8 \\
$\begin{array}{l}\text { Small } \\
\text { deformity }\end{array}$ & Atresia & Normal & 4 & 4 \\
\hline
\end{tabular}

Table 4. Type and number of performed operations

TYPE OF OPERATION

Combined reconstruction of middle and

external ear with harvesting of costal

NUMBER

cartilage

Combined reconstruction of middle ear

without cartilage graft

Stage II of operation

Stage III of operation

Reoperation 
Table 4. Results of reconstruction of combined external and middle ear malformations

\begin{tabular}{lcc}
\multicolumn{1}{c}{ RESULTS OF OPERATIONS } & \multicolumn{2}{c}{ NUMBER } \\
\hline AB gap 21-30 dB & No & $\%$ \\
AB gap 31-40 dB & 9 & 53,0 \\
AB gap 41-50 dB & 4 & 23,5 \\
Meatal stenosis & 4 & 23,5 \\
Complete meatal obliteration & 5 & 29,4 \\
Necrosis of cartilage transplant & 1 & 5,9 \\
\hline
\end{tabular}

In all cases with mayor malformations (mictoria gradus III, meatal atresia and middle ear malformation) we found fusion of malleus and incus with their fixation to atretic plate. Stapes was unchanged, but with significantly reduced visibility during surgery. Removal of atretic plate enabled ossicular mobility and adequate function. In all the cases mobile ossicular chain was achieved (figure 1-5).
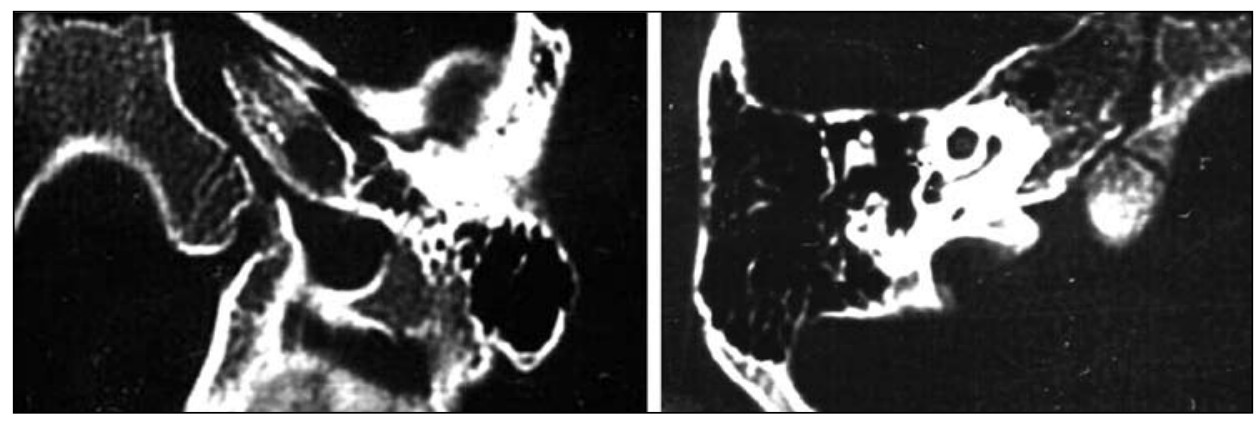

Figure 1. CT of congenital atresia: left atretic plate, right well developed middle ear space
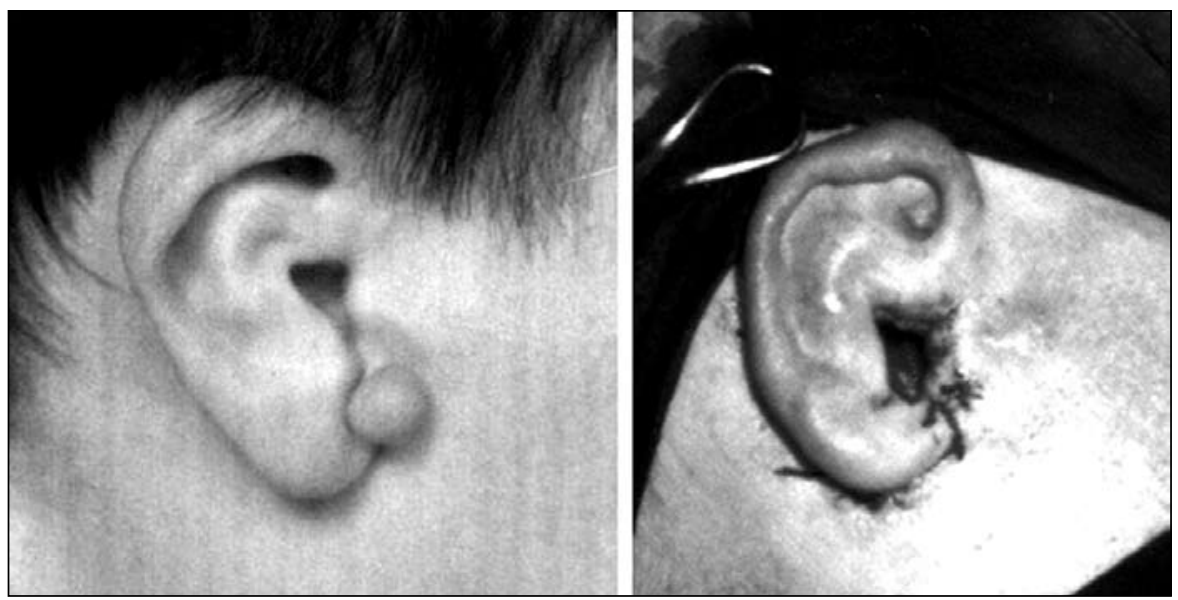

Figure 2. Reconstruction of atresia and minor auricular malformation
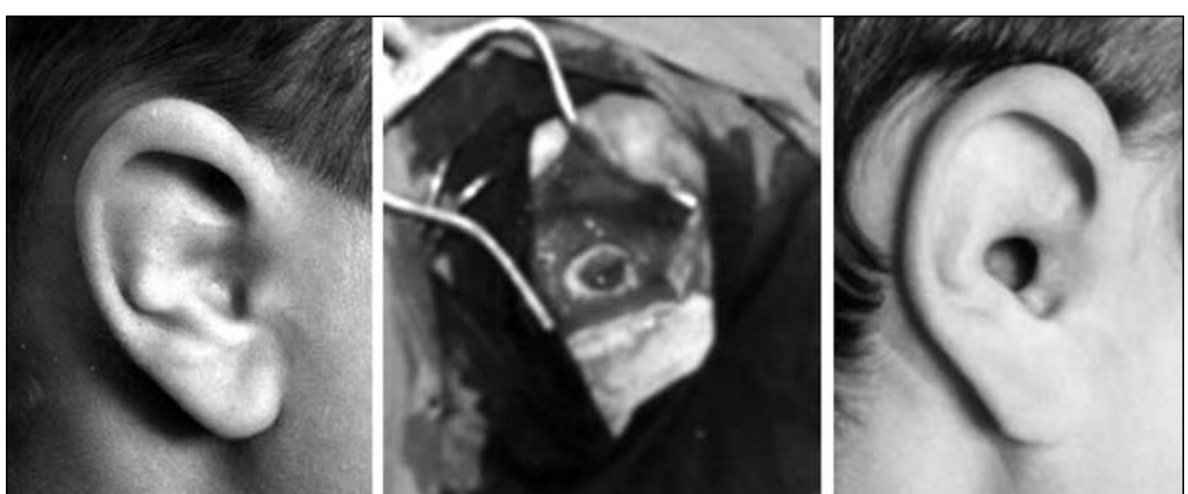

Figure 3. Atresia repair 

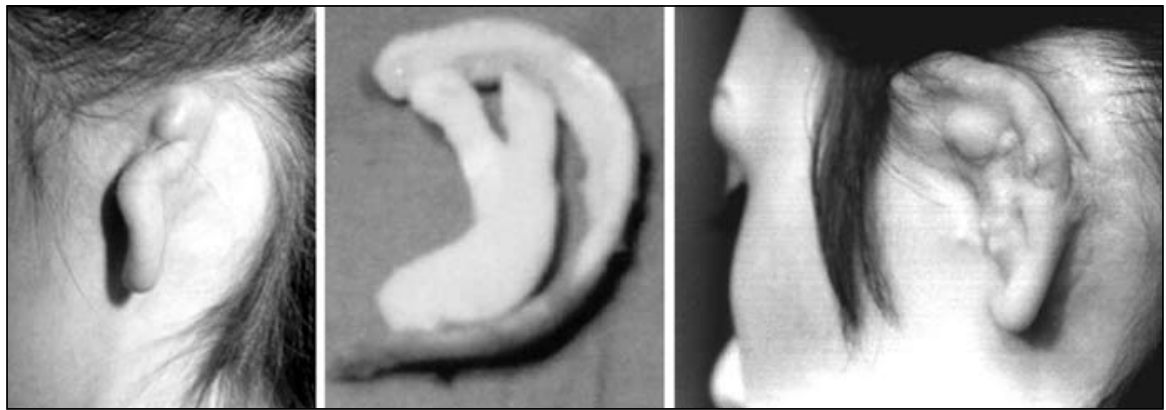

Figure 4. Reconstruction of artesian and major auricular malformation using cartilage transplant

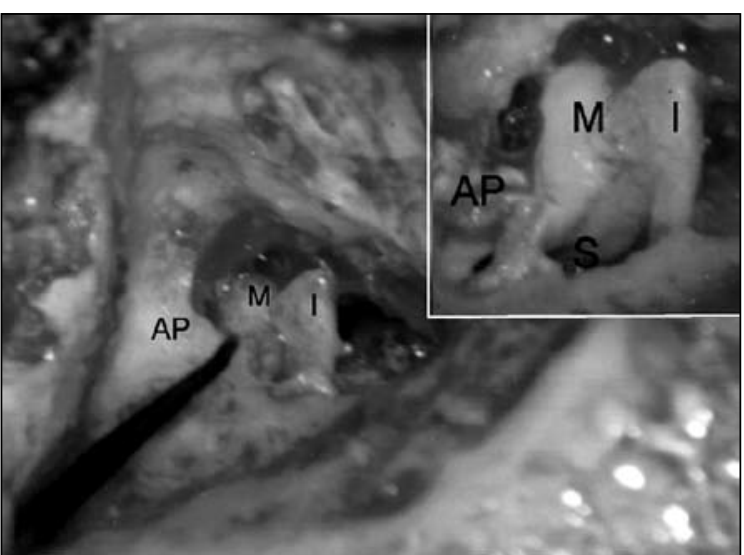

Figure 5. Intraoperative view on malformed ossicles (M-malleus, I-incus, S-stapes) and atretic plate (AP)
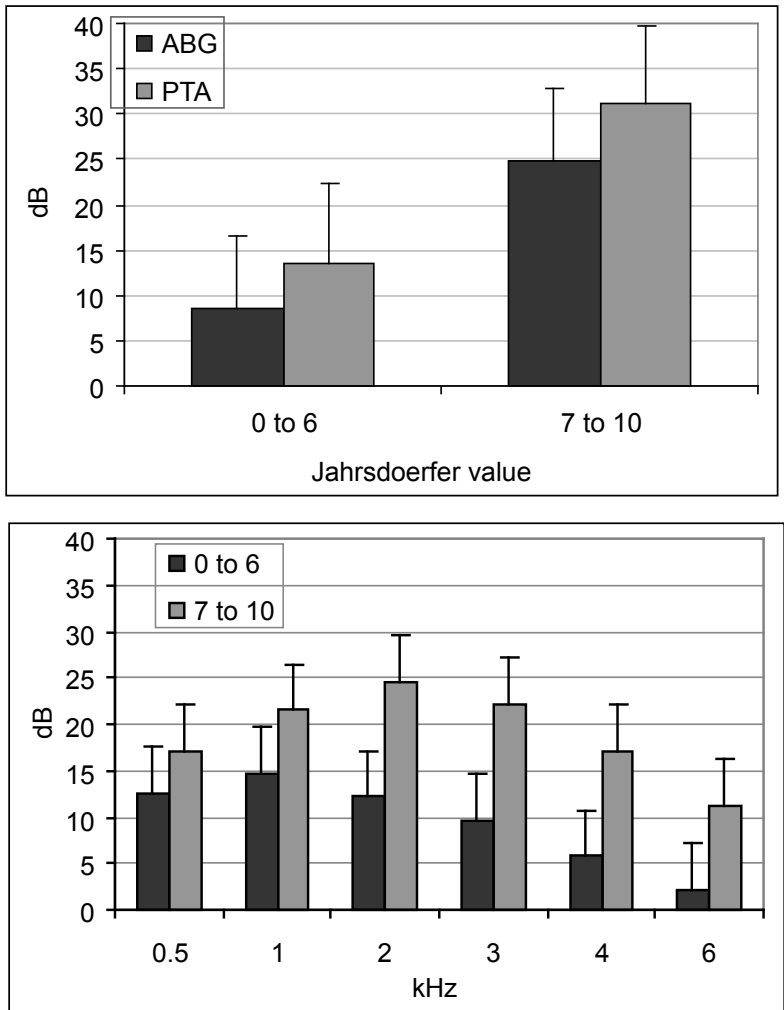

Figure $6 \mathbf{a}$ and $\mathbf{6 b}$. Postoperative audiometry according to Jahrsdoerfer classification $(A B G=$ air bone gap,

$\mathrm{PTA}=$ pure tone average, and haering gain for different frequencies in $\mathrm{kHz}$ )
In one case postoperative partial facial palsy was noticed, but the resolution was spontaneous. Sensorineural hearing loss and postoperative otorrhea were universally absent.

The degree of external ear deformity correlated with deformity of middle ear and results of operation. So, in cases of mayor auricular deformities big malformations of ossicles, reduced pneumatisation and facial malformations were always present. Audiological results were also worse.

Postoperative audiometry showed hearing threshold under $30 \mathrm{~dB}$ in $66,9 \%$ of patients, while in others it was 30-40 dB. Residual AB gap of $20 \mathrm{~dB}$ average was always present.

\section{Discussion}

Mayor congenital malformations of external ear have significant impact on psychosocial development. It is frequently combined with middle ear malformation causing consequent hearing loss and defect in speech development when it is bilateral. This demands early analysis of condition and further staged reconstruction.

Because of possible congenital cholesteatoma it is not generally accepted that only bilateral cases should be operated. Patients and parents opinion should be carefully taken in account. The ideal condition for operation is absence of facial deformities and adequate middle ear space. For bilateral atresia bone anchored hearing aids are used starting from the $4^{\text {th }}$ month, and operation of the first ear is recommended in the $4^{\text {th }}$ year.

The most frequent surgical attitude is individual approach of plastic surgeon and otosurgeon. Each of them wants to do his part of operation first, and he is usually concerned only for his part of work. ${ }^{3-5}$

The essence of reconstruction of malformed auricle is obtaining of sufficient aesthetic result with maximally reduced number of operations and avoidance of possible complications. Before the operation the parents should get precise informations concerning the type and possible results of planned surgical interventions. 
Proper selection of patients with combined external and middle ear malformations is necessary. Audiometry and auditory evoked potentials are needed for determination of status of conductive and sensorineural part and possibility of surgical reconstruction. Computerised tomography of temporal bone is performed before the operation in order to define the shape of cochlear and vestibular part of labyrinth, size of middle ear space, condition of auditory ossicles and course of facial nerve. It is especially important when suspicion on congenital cholesteatoma is present. If unilateral atresia is found together with extremely reduced pneumatic spaces $(50 \%$ smaller than contralateral healthy side), without visible ossicles operation is not recommended because of poor chance for success. Besides this, cochlear hypoplasia with enlarged vestibule and lateral semicircular canal indicates on possible pathologic communication of perilymph and CSF. ${ }^{3,6}$

Congenital aural atresia is a complex problem demanding multiple professional team in order to obtain adequate result. Systematic approach from the beginning to the end is demanded. Knowledge of anatomical characteristics of temporal bone and possible types of malformations affecting delicate structures is needed. Creating of proper external meatus, aerated middle ear space with functioning conductive system, preservation of facial nerve and inner ear are primary surgical demands. ${ }^{2,6}$

Surgical approach for repairment of atresia can be transmastoid or anterior. In mastoid approach sinodural angle is identified first, than antrum, facial recess and ossicular chain, with final removal of atretic plate. Contrary to this, anterior route involves elimination of bone between temporomandibular joint and middle cranial fossa, while surgical work in mastoid is only partial. This approach creates smaller operative cavity, and thus prevents accumulation of keratin and reduces the need for local therapy. Besides this, manipulation around facial nerve is significantly avoided. In cases of bigger ossicular deformities autotransplant of cortical bone, or other reconstructive materials can be used. ${ }^{8}$

Audiologic results of combined reconstruction are unequally reported. The success depends on the degree of deformity of external and middle ear.

Potential complications of staged reconstruction should not be overlooked. They include: skin necrosis over cartilaginous transplant, infection of cartilage, necrosis of lobulus, hypertrophic scar and meatal restenosis. Meatal restenosis is the most frequent postoperative problem.

\section{Conclusions}

Congenital combined malformations of external and middle ear have important psychological consequences on individual development. Affection of middle ear causes conductive hearing loss and delayed speech development when bilateral. Early diagnosis using BERA and CT is needed with subsequent surgical reconstruction. Proper selection of patients can result in hearing threshold up to $30 \mathrm{~dB}$ in over two thirds of patients. Modified surgical reconstruction with reduced number of operations and one surgeon performing all the interventions gives good aesthetic and functional results with very low complication rate.

\section{References:}

1. Lambert PR: MAJOR CONGENITAL EAR MALFORMATIONS: SURGICAL MANAGEMENT AND RESULTS. Ann Otol Rhinol Laryngol. 1988; 97: 641-649.

2. De la Cruz A, Linthicum FH Jr, Luxford WM: CONGENITAL ATRESIA OF THE EXTERNAL AUDITORY CANAL. Laryngoscope, 1985; 95: 421-427.

3. Aguilar EF: AURICULAR RECONSTRUCTION OF CONGENITAL MICROTIA (GRADE III). Laryngoscope. 1996; 106: 1-26.

4. Bauer BS: RECONSTRUCTION OF THE MICROTIC EAR. J Pediatr Surg. 1984; 19: 440-445.

5. Brent B: RECONSTRUCTION OF AURICLE, in McCarthy JG: Plastic Surgery. Philadelphia, WB Saunders Co, 1990: 2094-2152.
6. Yanai A, Fukuda O, Yamada A: PROBLEMS ENCOUNTERED IN CONTOURING A RECONSTRUCTED EAR OF AUTOGENOUS CARTILAGE. Plast Raconstr Surg. 1985; 75: 185-190.

7. Jahrsdoerfer RA, Yeakley JW, Agiular EA: GRADING SYSTEM FOR THE SELECTION OF PATIENTS WITH CONGENITAL AURAL ATRESIA. Am J Otol. 1992; 13: 6-12.

8. Kesselring UK, de Goumoens R: TEAM APPROACH TO TOTAL AURICULAR RECONSTRUCTION. Ann Plast Surg. 1991; 26: 299305 .

\section{Address:}

Milan Stankovic, MD

Pustorecka 33, 18000 Nis, Serbia

Tel. +38118520 595,

E-mail:milan.orl@bankerinter.net 